\title{
Retrospective, observational, cross-sectional study of detection of recurrent Barrett's esophagus and dysplasia in post-ablation patients with adjunctive use of wide-area transepithelial sample (WATS-3D)
}

\author{
Hala Fatima a, Maryiam Wajida, Nour Hamade ${ }^{a}$, Yan Han ${ }^{b}$, William Kesslera, John Dewitt ${ }^{a}$, Douglas Rex \\ Thomas Imperiale ${ }^{a}$
}

Indiana University School of Medicine, USA

\begin{abstract}
Background Barrett's esophagus (BE) and dysplasia are often missed by Seattle protocol biopsies (SPB). Wide-area transepithelial sampling with 3-dimensional computer-assisted analysis (WATS3D) with SPB improves detection in treatment-naïve patients. We aimed to determine to what extent WATS-3D adds to SPB in the detection of non-dysplastic BE (NDBE) and dysplasia in patients undergoing post-endoscopic eradication therapy (EET).

Methods This retrospective, observational, cross-sectional study included patients who presented for post-EET surveillance with SPB and WATS-3D sampling from April 2019 to February 2020. BE patients with no previous EET were excluded. For the outcomes of NDBE and any dysplastic/ neoplastic finding, we calculated both relative and absolute increases in yield by WATS-3D over SBP.

Results In 78 patients [mean age $68 \pm 10.4$ years, 66 (84.6\%) male], the prevalence of NDBE, any dysplastic/neoplastic finding, and any abnormality (NDBE or dysplasia/neoplasia) were $53.85 \%$, $10.26 \%$, and $55.13 \%$. The absolute increase in yield of NDBE with WATS-3D over SPB was $26.9 \%$ (95\% confidence interval [CI] 17.95-37.18\%), with the number needed to treat (NNT) 3.71 (95\%CI 2.69-5.57) and a relative increase in yield of 100\% (95\%CI 53.33-188.25\%). For dysplasia/neoplasia, the absolute increase in yield was 6.4\% (95\%CI 1.28-12.82\%), NNT 15.6 (95\%CI 7.8-78.0), and relative increase of $167 \%$ ( $95 \% \mathrm{CI} 33.33 \%$-infinity). For any abnormal finding, the absolute increase in yield was $26.9 \%$ (95\%CI 16.67-37.18\%), NNT 3.71 (95\%CI 2.69-6.00), and relative increase in yield 95\% (95\%CI 50-176.92\%).
\end{abstract}

Conclusions WATS-3D with SPB improves the detection of residual/recurrent BE and dysplasia in post-ablation BE. However, randomized controlled trials are needed to validate these findings.

Keywords Barrett's esophagus, Seattle protocol, dysplasia, detection, post-endoscopic eradication therapy

Ann Gastroenterol 2022; 35 (X): 1-7

a'Department of Internal Medicine, Division of Gastroenterology (Hala Fatima, Maryiam Wajid, Nour Hamade, William Kessler, John Dewitt, Douglas Rex, Thomas Imperiale); 'Department of Biostatistics and Health Data Science (Yan Han), Indiana University School of Medicine

Conflict of Interest: John Dewitt: Research grant: Vyaire Medical; Consultant: Boston Scientific and Cook Medical.

Douglas Rex: Consultant: Olympus Corporation, Boston Scientific, Aries Pharmaceutical, Braintree Laboratories, Lumendi Ltd, Norgine, Endokey, GI Supply, Covidian/Medtronic. Research Support: EndoAid, Olympus Corporation, Medivators, Erbe USA Inc, Braintree Laboratories. Shareholder: Satisfai Health

Correspondence to: Name Hala Fatima MD, 702 Rotary Circle, Suite 225, Indianapolis, IN 46202, USA, e-mail: hafatima@iu.edu

Received 24 September 2021; accepted 25 November 2021; published online 7 January 2022

DOI: https://doi.org/10.20524/aog.2022.0693

\section{Introduction}

Esophageal adenocarcinoma (EAC) is associated with a 5 -year survival rate of $15-34 \%$ [1], and Barrett's esophagus (BE) is its only known precursor [2]. Current guidelines recommend endoscopic eradication therapy (EET) for selected patients with low-grade dysplasia (LGD), as well as those with high-grade dysplasia (HGD) and T1a EAC/intramucosal adenocarcinoma (ImCA) [3]. EET is also used to treat selected patients with non-dysplastic BE (NDBE): e.g., those with a family history of esophageal cancer or high anxiety associated with the diagnosis of BE, or reflecting the surgeon's preference before fundoplication surgery [4]. However, recurrence of BE after these therapies is possible, and continued surveillance of patients is thus recommended. The recurrence rate of NDBE 
is $4.8 \% / 100$ patient-years (PY), and dysplasia is $2.0 \% / 100$ PY after EET [5]. Current surveillance for residual BE and dysplasia involves esophagogastroduodenoscopy (EGD) with Seattle protocol biopsy (SPB), which consists of taking 4-quadrant biopsies every $1 \mathrm{~cm}$ in areas of $\mathrm{BE}$ in patients with known dysplasia [6]. Almost $80 \%$ of all biopsy samples are adequate to evaluate for subsquamous intestinal metaplasia in post-ablation patients [7]. However, SPB is time-consuming, especially in patients with long-segment BE [8], and has low protocol adherence by endoscopists (from $85 \%$ in 2013 to $81 \%$ in 2017) [9]. In addition, sampling errors may occur, as this technique collects samples from only $\sim 5 \%$ of the total area of BE, and may miss early cancer or dysplasia [10]. Given these limitations, there is a clear need for effective and easier surveillance techniques for $\mathrm{BE}$.

Wide-area transepithelial sampling with computer-assisted 3-dimensional analysis (WATS-3D) is a novel technique (CDx diagnostics, Suffern, NY) that uses a stiff $1.8-\mathrm{mm}$ brush, passed through the endoscope channel to sample a wide area of tissue [11]. The brush is abrasive and samples the full thickness of the epithelium. The specimen is then analyzed using an artificial intelligence neural network with 3D image processing to identify abnormal cells. This method allows quicker sampling and obtains transepithelial tissue, which may minimize the risk of missed lesions. Previous studies in treatment-naïve patients have shown $16 \%$ and $62 \%$ higher rates of $\mathrm{BE}$ and dysplasia detection [12], respectively, when WATS-3D is used in conjunction with 4-quadrant biopsies and targeted biopsies [13-16]. However, no study has reported its use in patients with previous EET, except for 1 in abstract form [17]. Therefore, to demonstrate the effectiveness of WATS-3D in this population, the first step is to establish its role as an adjunct to SPB.

This study's primary endpoint was to compare intestinal metaplasia and dysplasia detection rates between WATS-3D and SPB in patients who underwent EET for BE. The secondary outcomes were to determine the added yield with WATS-3D in the post-ablation setting and to evaluate the agreement of findings between WATS-3D and SPB.

\section{Patients and methods}

Our study was a retrospective, observational, crosssectional study. CDx Diagnostics (Suffern, NY) had no role in the study design, data collection, interpretation, or manuscript preparation. This study was approved by the institutional review board of Indiana University Purdue University at Indianapolis (IRB \# 2001781987).

\section{Patient selection}

Patients over 18 years who presented to the Indiana University endoscopy suite post EET for BE with LGD, HGD, ImCA or EAC, and had undergone SPB and WATS$3 \mathrm{D}$ sampling, were included in the study. All patients showed complete eradication of intestinal metaplasia on 2 consecutive surveillance mapping biopsies, 3-6 months apart. These patients had previously undergone endoscopic interventions, including endoscopic mucosal resection (EMR), radiofrequency ablation (RFA), cryotherapy, argon plasma coagulation (APC), or a combination of ablative therapies. All patients had visually absent BE on surveillance exam. Any patients with visible abnormalities on EGD that required target biopsies were excluded. Surveillance included both SPB and WATS-3D sampling. The study duration was from April 2019 to February 2020. Patients with no prior EET were excluded.

\section{EGD and tissue sampling}

Four experienced staff endoscopists performed esophagogastroduodenoscopy in the standard fashion. The mucosal inspection was performed using high-definition white light endoscopes with digital chromoendoscopy. Biopsies were taken using cold forceps per the SPB every $1 \mathrm{~cm}$ over the original pre-ablation extent of $\mathrm{BE}$, and from the cardia just distal to the squamocolumnar junction (SCJ). Immediately after the SPB, WATS-3D brushing was performed over the same area of esophageal mucosa and cardia. One of the 4 endoscopists (16 patients) performed biopsy sampling and WATS-3D brushing only at the level of the SCJ and cardia, instead of the whole length of the neo-squamous epithelium. If there were any recurrent visible abnormalities, those patients were excluded from the analysis. Either ablation or targeted biopsies were performed in those cases.

The WATS-3D kit comes with 2 brushes, inserted through the biopsy channel to collect a cellular sample from the mucosa. The first brush is rubbed against the epithelial surface of the esophageal mucosa and cardia with both back-and-forth and circumferential motion. The cells from the brush are smeared on a slide and fixed. The brush is then clipped and placed in the enclosed jar for cell block preparation. The second brush is used to collect samples over the same segment of mucosa in the same way as the first, and is clipped and placed in the same jar. The jar is then submitted along with the slide to the lab for interpretation. For patients with long-segment BE, a separate kit was used for every $5 \mathrm{~cm}$. All WATS-3D samples were packaged and sent to CDx diagnostics in Suffern, NY, for analysis.

\section{Pathology interpretation}

SPBs were reviewed at the Indiana University pathology lab by a gastrointestinal pathologist and confirmed by a second pathologist. These pathologists were blinded to the WATS-3D results. Analysis of WATS-3D specimens employs a computerized 3D image analysis system. Neural networks then examine the $3 \mathrm{D}$ computer-synthesized images to produce a high-resolution video. The slides from the WATS-3D samples were analyzed at CDx diagnostics (Suffern, NY) by pathologists. The CDx pathologists were blinded to the SPB results. The 
pathology results were recorded as the presence of LGD, HGD, ImCA, or EAC or any combination of these findings, termed as "any abnormality."

\section{Statistical analysis}

Demographic, endoscopic, and pathology data were collected and de-identified before analysis. Clinical and demographic characteristics were summarized as the mean \pm standard deviation for continuous variables and frequencies, and as percentages for categorical variables. The metric used for evaluating the clinical utility of WATS-3D is "added yield," defined as:

$$
\text { Added yield }=\frac{\text { (Outcome detected by WATS alone })}{\begin{array}{l}
{[\text { (Outcome detected by WATS }+ \text { SBP })} \\
+(\text { Outcome detected by SPB alone })]
\end{array}}
$$

We recorded the crude difference in yield of detecting BE using WATS-3D as an adjunctive technique compared to SPB, and McNemar's test to compare the agreement of the measurements between SBP and WATS-3D brushing for detecting any abnormality, namely BE, LGD, HGD, and ImCA. A P-value of $<0.05$ was considered significant. In addition, we used Kappa statistics to measure the agreement between the SPB and WATS-3D results. We used SAS v9.4 for all analyses.

\section{Results}

\section{Patient characteristics}

The study included a total of 78 patients, with a mean age of $68 \pm 10.4$ years, of whom $77(98.7 \%)$ were white, and $66(84.6 \%)$ were men. Patient demographics are shown in Table 1 . The majority were current or previous smokers (59\%). The mean length of $\mathrm{BE}$ was $5 \pm 4.77 \mathrm{~cm}$, and hiatal hernia was present in $91 \%$; the mean hiatal hernia size was $3.6 \pm 2.13 \mathrm{~cm}$. Previous therapies included EMR (61.5\%), RFA (73\%), cryotherapy (17.9\%), and APC (46\%). There were no adverse events (AE) reported with SPB or WATS-3D sampling.

\section{Cases detected by WATS-3D vs. SPB}

Prevalence of NDBE, any dysplastic/neoplastic finding, and any abnormality were $53.85 \%(n=42), 10.26 \%(n=8)$ and $55.13 \%(n=43)$, respectively. NDBE was detected in 9 patients with SPB but missed on WATS-3D and in 21 patients with WATS-3D whose SPB results were negative. Similarly, LGD was detected in 1 patient with SPB that was missed on WATS$3 \mathrm{D}$, whereas WATS-3D detected LGD in 6 patients missed with SPB (Table 2).

To evaluate the clinical utility of WATS, we reported the "added yield" of WATS-3D to SPB for detection of NDBE

\begin{tabular}{|c|c|}
\hline Variable & Mean \pm SD/Frequency (\%) \\
\hline Age (years) & $67.83 \pm 10.42$ \\
\hline Weight (kg) & $95.00 \pm 20.93$ \\
\hline Height $(\mathrm{cm})$ & $176.25 \pm 9.13$ \\
\hline BMI $\left(\mathrm{kg} / \mathrm{m}^{2}\right)$ & $30.55 \pm 6.35$ \\
\hline $\begin{array}{l}\text { Sex } \\
\quad \text { Female } \\
\text { Male }\end{array}$ & $\begin{array}{l}12(15.4) \\
66(84.6)\end{array}$ \\
\hline $\begin{array}{l}\text { Ethnicity } \\
\text { White } \\
\text { African American }\end{array}$ & $\begin{array}{c}77(98.7) \\
1(1.3)\end{array}$ \\
\hline $\begin{array}{l}\text { Smoking } \\
\text { Non-smoker } \\
\text { Past smoker } \\
\text { Current smoker } \\
\text { Unknown }\end{array}$ & $\begin{array}{c}31(39.7) \\
40(51.3) \\
6(7.7) \\
1(1.3)\end{array}$ \\
\hline $\begin{array}{l}\text { Alcohol use } \\
\text { Yes } \\
\text { No } \\
\text { Unknown }\end{array}$ & $\begin{array}{c}45(57.7) \\
30(38.5) \\
3(3.8)\end{array}$ \\
\hline Original length of Barrett's (cm) & $5.14 \pm 4.77$ \\
\hline Presence of hiatal hernia & $71(91)$ \\
\hline Hiatal hernia size $(\mathrm{cm})$ & $3.60 \pm 2.13$ \\
\hline $\begin{array}{l}\text { Highest degree of dysplasia prior t } \\
\text { ablation } \\
\text { NDBE } \\
\text { LGD } \\
\text { HGD } \\
\text { ImCA } \\
\text { EAC }\end{array}$ & $\begin{array}{c}13(16.7) \\
16(20.5) \\
34(43.6) \\
10(12.8) \\
5(6.4)\end{array}$ \\
\hline $\begin{array}{l}\text { Previous endoscopic therapy } \\
\text { Endoscopic mucosal resection } \\
\text { Radiofrequency ablation } \\
\text { Cryotherapy } \\
\text { Argon plasma coagulation }\end{array}$ & $\begin{array}{c}48(61.5) \\
57(73.1) \\
14(18) \\
36(46.2)\end{array}$ \\
\hline
\end{tabular}

Table 1 Baseline clinical and demographic characteristics

kg, kilogram; cm, centimeter; BMI, body mass index; NDBE, non-dysplastic Barrett's esophagus; LGD, low-grade dysplasia; HGD, high-grade dysplasia; ImCA, intramucosal adenocarcinoma; EAC, esophageal adenocarcinoma with at least submucosal invasion

BE, dysplasia, or ImCA with WATS-3D. The added yield of NDBE with WATS-3D over SPB was $26.9 \%$ (95\% confidence interval [CI] 17.95-37.18\%), with a number needed to treat (NNT) 3.71 (95\%CI 2.69-5.57) and a relative increase in yield of $100 \%$ (95\%CI 53.33-188.25\%). For dysplasia/neoplasia, the absolute increase in yield was $6.4 \%$ (95\% CI 1.28-12.82\%), NNT 15.6 (95\%CI 7.8-78.0), and the relative increase was $167 \%$ (95\%CI 33.33\%-infinity). For any abnormal finding, the absolute increase in yield was $26.9 \%$ (95\%CI $16.67-37.18 \%$ ), NNT 3.71 (95\%CI 2.69-6.00), and the relative increase was 95\% (95\%CI 50-176.92\%) (Table 3).

Furthermore, among the additional instances of dysplasia/ neoplasia found by WATS-3D, 4 cases were upgraded from SPB by WATS-3D: 1 from HGD to ImCA and 3 from NDBE to LGD. This was a total absolute increase of $10.3 \%(8 / 78)$ for a 
Table 2 Proportion of detection by WATS-3D versus Seattle Protocol biopsies

\begin{tabular}{|c|c|c|c|c|c|}
\hline Positive results & NDBE n (\%) & LGD n (\%) & HGD n (\%) & $\operatorname{ImCA} \mathrm{n}(\%)$ & Any abnormality $\mathrm{n}(\%)$ \\
\hline WATS-3D & $33(78.6)$ & $6(85.7)$ & $2(66.7)$ & $1(100)$ & $34(79.1)$ \\
\hline Seattle protocol & $21(50)$ & $1(14.3)$ & $2(66.7)$ & $0(0)$ & $22(51.2)$ \\
\hline Both & $12(28.6)$ & $0(0)$ & $1(33.3)$ & $0(0)$ & $13(30.2)$ \\
\hline Total & 42 & 7 & 3 & 1 & 43 \\
\hline
\end{tabular}

Table 3 Detection of NDBE, dysplasia and neoplasia $(n=78)$

\begin{tabular}{|c|c|c|c|c|c|c|}
\hline \multirow[t]{2}{*}{ Abnormality } & \multicolumn{2}{|c|}{ Increased detection of NDBE } & \multicolumn{2}{|c|}{$\begin{array}{l}\text { Increased detection of dysplastic } \\
\mathrm{BE}\end{array}$} & \multicolumn{2}{|c|}{$\begin{array}{l}\text { Increased detection of } \\
\text { any abnormality }\end{array}$} \\
\hline & WATS-3D negative & WATS-3D positive & WATS-3D negative & $\begin{array}{l}\text { WATS-3D } \\
\text { positive }\end{array}$ & $\begin{array}{l}\text { WATS-3D } \\
\text { negative }\end{array}$ & $\begin{array}{l}\text { WATS-3D } \\
\text { positive }\end{array}$ \\
\hline SPB negative & 36 & 21 & 70 & 5 & 35 & 21 \\
\hline \multirow[t]{2}{*}{ SPB positive } & 9 & 12 & 2 & 1 & 9 & 13 \\
\hline & \multicolumn{2}{|l|}{ Absolute difference } & Absolute increase & $\begin{array}{l}\text { Added } \\
\text { yield }\end{array}$ & $\begin{array}{l}\text { Relative } \\
\text { increase }\end{array}$ & NNT \\
\hline NDBE & \multicolumn{2}{|l|}{12} & $26.9 \%$ & 1 & $100 \%$ & 3.71 \\
\hline Dysplasia or neoplasia & \multicolumn{2}{|l|}{3} & $6.4 \%$ & 1.67 & $167 \%$ & 15.6 \\
\hline Any abnormality & \multicolumn{2}{|l|}{12} & $26.9 \%$ & 0.95 & $95 \%$ & 3.71 \\
\hline \multicolumn{7}{|l|}{ Added yield= b/(c+d) } \\
\hline \multicolumn{7}{|c|}{ Relative increase $=b /(c+d) \times 100$} \\
\hline \multicolumn{7}{|c|}{ Absolute increase $($ added detection $)=b /(a+b+c+d)$} \\
\hline \multicolumn{7}{|l|}{$\mathrm{NNT}=(\mathrm{a}+\mathrm{b}+\mathrm{c}+\mathrm{d}) / \mathrm{b}$} \\
\hline \multicolumn{7}{|c|}{ Absolute difference of WATS over SPB $=(b+d)-(c+d)$} \\
\hline
\end{tabular}

new diagnosis of dysplasia and $15.4 \%(12 / 78)$ when accounting for an upgrade of dysplasia grade (Table 4). In 2 patients, the diagnosis from WATS-3D was upgraded by SPB: one from NDBE to HGD and another from NDBE to LGD.

\section{Agreement between WATS and SPB}

Agreement of findings between WATS-3D and SPB was also measured (Table 5). There was no agreement between SPB and WATS-3D results for NDBE $(\mathrm{P}=0.0285)$. In addition, there was insufficient evidence to show any agreement between the 2 tests for LGD and HGD.

\section{Discussion}

Our single-center cohort of post-ablation patients demonstrates that the use of WATS-3D in addition to SP increases detection of NDBE, LGD, HGD, and ImCA on surveillance with a combined added yield of $95 \%$. This
Table 4 Adjunctive yield of WATS-3D

\begin{tabular}{lcccc}
$\begin{array}{l}\text { Dysplasia } \\
\text { grade }\end{array}$ & $\begin{array}{c}\text { New cases } \\
\text { of dysplasia }\end{array}$ & $\begin{array}{c}\text { Upgraded cases } \\
\text { of dysplasia }\end{array}$ & Total & \% of cases \\
\hline LGD & 6 & 3 & 9 & 11.5 \\
HGD & 1 & 0 & 1 & 1.3 \\
\hline ImCA & 1 & 1 & 2 & 2.6 \\
Total & 8 & 4 & 12 & 15.4 \\
\hline
\end{tabular}

WATS-3D, wide-area trans-epithelial sampling; LGD, low-grade dysplasia; HGD, high-grade dysplasia; ImCA, intramucosal adenocarcinoma

increased yield was 100\% for NDBE and 167\% for dysplasia/ neoplasia.

The guidelines of the American College of Gastroenterologists indicate that the accuracy of SPB is in the range of $35-68 \%$ in patients presenting for BE screening [18]. The wide-area approach of WATS-3D can be used to minimize this sampling error and missed lesions. Several studies have reported the higher yield of WATS-3D in screening and surveillance cohorts of BE patients [19]. Smith et al and Johanson et al showed a $19.9 \%$ and $39.8 \%$ increase in detection of $\mathrm{BE}$, respectively, with WATS in patients undergoing $\mathrm{BE}$ 
Table 5 Agreement between SP forceps biopsy results and WATS-3D results

\begin{tabular}{|c|c|c|c|c|c|c|c|}
\hline \multirow{2}{*}{$\begin{array}{l}\text { Compare standard biopsies } \\
\text { to WATS-3D brushing for } \\
\text { detecting }\end{array}$} & \multirow{2}{*}{$\begin{array}{c}\text { SPB } \\
\text { positive }\end{array}$} & \multirow{2}{*}{$\begin{array}{c}\text { WATS-3D } \\
\text { positive }\end{array}$} & \multirow{2}{*}{$\begin{array}{l}\text { SBP \& } \\
\text { WATS-3D } \\
\text { positive }\end{array}$} & \multirow{2}{*}{$\begin{array}{l}\text { P-value of } \\
\text { McNemar's } \\
\text { test }\end{array}$} & \multicolumn{3}{|c|}{ Simple kappa coefficient } \\
\hline & & & & & Estimate & Standard error & $95 \%$ CI \\
\hline NDBE & 21 & 33 & 12 & 0.0285 & 0.17 & 0.11 & {$[-0.04,0.38]$} \\
\hline LGD & 1 & 6 & 0 & $0.1250^{*}$ & -0.02 & 0.02 & {$[-0.06,0.02]$} \\
\hline HGD & 2 & 2 & 1 & $1.0000^{*}$ & 0.49 & 0.31 & {$[-0.12,1.00]$} \\
\hline $\operatorname{ImCA}$ & 0 & 1 & 0 & N/A & N/A & N/A & N/A \\
\hline
\end{tabular}

*Indicates the exact McNemar's test

WATS-3D, Wide area trans-epithelial sampling; CI, confidence interval; NDBE, non-dysplastic Barrett's esophagus; LGD, low-grade dysplasia; HGD, high-grade dysplasia; ImCA, intramucosal adenocarcinoma; SBP, Seattle protocol biopsies

screening and surveillance $[14,15]$. The increase in dysplasia detection was $42 \%$ with the addition of WATS-3D over SBP per Anandasabapathy et al [13], and the yield of HGD/EAC was 4 times higher per Vennalaganti et al in patients undergoing $\mathrm{BE}$ surveillance [16]. A meta-analysis of 12 studies of BE surveillance showed a $16-62 \%$ increase in the detection rate of BE with a marginal $2 \%$ absolute increase in the detection of dysplasia [12]. A recent meta-analysis showed that WATS$3 \mathrm{D}$ increased the absolute yield of dysplastic BE (all grades including indeterminate dysplasia) and HGD/EAC over conventional biopsies by $7.2 \%$ and $2.1 \%$, respectively [20]. While these studies show promising results for the use of WATS-3D in the diagnosis and surveillance of BE, no published study has yet reported on the use of WATS-3D in the post-ablation setting.

An important fact pointed out in the meta-analysis by Codipilly et al is that WATS-3D can be negative in $62.5 \%$ of cases where SPB detected dysplasia [20]. Therefore, based on current literature, WATS-3D can only be used as an adjunctive modality to SPB for BE surveillance. In addition, the results are discordant because of the differences in sampling technique and criteria for dysplasia assessment in the 2 approaches. The deeper sampling creates the possibility of overdiagnosis with WATS-3D, with studies indicating that only $30-35 \%$ of instances of WATS-3D detected dysplasia were confirmed on subsequent biopsies $[13,16]$.

Our study showed $54 \%$ and $10 \%$ prevalence for NDBE and dysplasia/neoplasia, respectively, post-EET, higher than other studies. One reason could be that, in most cases, we biopsied and brushed the neosquamous epithelium based on the original extent of pre-treatment Barrett's, thus increasing the detection rate.

While there have been no large-scale studies to evaluate the cost-effectiveness of the addition of WATS-3D to SPB, Singer et al have reported on an analytical model that found that screening with WATS-3D, when used with the SPB, was more cost-effective compared to SPB alone [21]. WATS-3D also seems to be a safe procedure, as a retrospective analysis performed by Docimo et al reported no AE with its use [22]. Our study showed no AEs associated with its use. Thus, with guideline support and favorable cost and safety profiles, it seems WATS-3D might be a promising tool for widespread use in BE sampling. Guidelines by the American Society of
Gastrointestinal Endoscopy recommend that WATS-3D be used as an adjunct to SPB in patients with BE. However, this was graded as a conditional recommendation based on lowquality evidence [3]. No recommendations exist for the use of WATS-3D in the post-EET population.

In our study, the increased yield of WATS-3D over SPB was more pronounced for the detection of dysplasia/neoplasia as compared to NDBE. The reason for these differences is unclear but may have to do with our population being high risk, given their prior diagnosis of dysplasia/neoplasia. Almost 20\% had a diagnosis of ImCA or EAC before ablation, and therefore had an higher risk of recurrence. Earlier detection of dysplasia in the post-EET population can allow for earlier endoscopic treatment; hence the importance of the higher yield of WATS$3 \mathrm{D}$ in this population. Given the greater yield of NDBE and dysplasia with WATS-3D in the post-EET population, its ease of use, the absence of associated AEs, and the opportunity for early detection and therapy, its routine use in surveillance could be recommended in the future as more data become available.

Mapping biopsies for BE surveillance in post-EET patients are limited by scar tissue from the previous ablation, in addition to the sampling error associated with 4-quadrant point biopsies. They are also relatively inefficient and time-consuming, leading to endoscopist fatigue and poor compliance [23]. Brushing for WATS-3D, on the other hand, is quick, with sampling for segments up to $5 \mathrm{~cm}$ with a single kit. In addition, it is easy to perform, as endoscopists are already familiar with brushings, with no training requirement.

The major strength of our study is a post-EET population that has not yet been studied for the efficacy of WATS-3D. Barrett's experts, who are well versed in routinely carrying out SPB and WATS-3D sampling, did the surveillance. In addition, WATS-3D and SPB were analyzed at different laboratories by different sets of pathologists who were blinded to the others' findings. The WATS-3D images were read based on standard pathologic criteria. Although the methodology in the study was not uniform-one endoscopist (16 patients) only performed sampling at the cardia and at the SCJ, which has the potential to introduce bias in the results-this is closer to actual clinical practice.

There are some limitations to this study. First, it included a small number of patients from one center and so was not 
adequately powered to reach significance. Second, it was based in a single center in a tertiary academic institution, and hence the results cannot be generalizable to community-based practices. Furthermore, this was a retrospective analysis, with the inherent limitations of that study design, while neither the Indiana University pathologists nor the WATS-3D pathologists were blinded to the baseline pathology findings. In addition, SPB was performed before WATS-3D in all cases, and one of the endoscopists sampled only SCJ and cardia. Finally, our study only looked at WATS-3D as an adjunct tool, but could not answer the question of whether WATS-3D can be used on its own as a substitute for SPB in the detection of BE in postablation patients.

Despite the above limitations, our study was able to show an added benefit from the use of WATS-3D as an adjunct to SPB. While SPB is efficacious in sampling targeted visible mucosal abnormalities with high localization specificity, it can miss lesions with a random sampling of other parts of the esophagus. In addition, outcomes data in BE suggests that histopathologic grade is the highest predictor for disease severity and dictates treatment. With increased detection of disease, better-informed decisions can be made about patients' management and treatment options.

In conclusion, the adjunctive use of WATS-3D with SPB can be a promising tool to increase detection of $\mathrm{BE}$ in post-ablation patients. However, more extensive, prospective, randomized studies are needed to further validate these findings in academic and community settings. In addition, whether WATS-3D has the potential to be used alone in the future will require further studies that compare the use of WATS in conjunction with SPB vs. SPB alone vs. WATS-3D alone, to address the question of whether WATS-3D can be a possible substitute for SPB in certain situations.

\section{Summary Box}

\section{What is already known:}

- Wide-area transepithelial sampling with 3-dimensional computer-assisted analysis (WATS3D) allows for quicker sampling as compared to Seattle protocol biopsies (SBP)

- WATS-3D used in conjunction with SBP is associated with a higher detection rate of $\mathrm{BE}$ and dysplasia in treatment-naïve patients

\section{What the new findings are:}

- WATS-3D with SPB improves the detection of residual/recurrent $\mathrm{BE}$ and dysplasia in postablation patients

- The absolute increase in yield with WATS-3D was $26.9 \%$, and the relative increase in yield was $95 \%$

- There were no adverse events related to the use of WATS-3D

\section{References}

1. Deng HY, Wang WP, Wang YC, et al. Neoadjuvant chemoradiotherapy or chemotherapy? A comprehensive systematic review and meta-analysis of the options for neoadjuvant therapy for treating oesophageal cancer. Eur J Cardiothorac Surg 2017;51:421-431.

2. Siegel RL, Miller KD, Jemal A. Cancer statistics, 2019. CA Cancer J Clin 2019;69:7-34.

3. Qumseya B, Sultan S, Bain P, et al; ASGE Standards of Practice Committee Chair. ASGE guideline on screening and surveillance of Barrett's esophagus. Gastrointest Endosc 2019;90:335-359.

4. Fleischer DE, Odze R, Overholt BF, et al. The case for endoscopic treatment of non-dysplastic and low-grade dysplastic Barrett's esophagus. Dig Dis Sci 2010;55:1918-1931.

5. Fujii-Lau LL, Cinnor B, Shaheen N, et al. Recurrence of intestinal metaplasia and early neoplasia after endoscopic eradication therapy for Barrett's esophagus: a systematic review and metaanalysis. Endosc Int Open 2017;5:E430-E449.

6. Evans JA, Early DS, Fukami N, et al; Standards of Practice Committee of the American Society for Gastrointestinal Endoscopy. The role of endoscopy in Barrett's esophagus and other premalignant conditions of the esophagus. Gastrointest Endosc 2012;76:1087-1094.

7. Shaheen NJ, Peery AF, Overholt BF, et al; AIM Dysplasia Investigators. Biopsy depth after radiofrequency ablation of dysplastic Barrett's esophagus. Gastrointest Endosc 2010;72:490-496.

8. Wani S, Williams JL, Komanduri S, Muthusamy VR, Shaheen NJ. Endoscopists systematically undersample patients with longsegment Barrett's esophagus: an analysis of biopsy sampling practices from a quality improvement registry. Gastrointest Endosc 2019;90:732-741.

9. Wani S, Williams JL, Komanduri S, Muthusamy VR, Shaheen NJ. Time trends in adherence to surveillance intervals and biopsy protocol among patients with Barrett's esophagus. Gastroenterology 2020;158:770-772.

10. Jankowski M, Wani S. Diagnostic and management implications of basic science advances in Barrett's esophagus. Curr Treat Options Gastroenterol 2015;13:16-29.

11. Thosani N, Abu Dayyeh BK, Sharma P, et al; ASGE Technology Committee. ASGE Technology Committee systematic review and meta-analysis assessing the ASGE preservation and incorporation of valuable endoscopic innovations thresholds for adopting real-time imaging-assisted endoscopic targeted biopsy during endoscopic surveillance of Barrett's esophagus. Gastrointest Endosc 2016;83:684-698.e7.

12. Suresh Kumar VC, Harne P, Patthipati VS, et al. Wide-area transepithelial sampling in adjunct to forceps biopsy increases the absolute detection rates of Barrett's oesophagus and oesophageal dysplasia: a meta-analysis and systematic review. BMJ Open Gastroenterol 2020;7:e000494.

13. Anandasabapathy S, Sontag S, Graham DY, et al. Computerassisted brush-biopsy analysis for the detection of dysplasia in a high-risk Barrett's esophagus surveillance population. Dig Dis Sci 2011;56:761-766.

14. Johanson JF, Frakes J, Eisen D; EndoCDx Collaborative Group. Computer-assisted analysis of abrasive transepithelial brush biopsies increases the effectiveness of esophageal screening: a multicenter prospective clinical trial by the EndoCDx Collaborative Group. Dig Dis Sci 2011;56:767-772.

15. Smith MS, Ikonomi E, Bhuta R, et al. Wide-area transepithelial sampling with computer-assisted 3-dimensional analysis (WATS) markedly improves detection of esophageal dysplasia and Barrett's esophagus: analysis from a prospective multicenter communitybased study. Dis Esophagus 2019;32:doy099. 
16. Vennalaganti PR, Kaul V, Wang KK, et al. Increased detection of Barrett's esophagus-associated neoplasia using wide-area transepithelial sampling: a multicenter, prospective, randomized trial. Gastrointest Endosc 2018;87:348-355.

17. Pagan AS, Diaz L, Arroyo Y, Bertran-Rodriguez C, Klocksieben F, Pena L. Wide area transepithelial sampling in adjunct to forceps biopsy in post treatment Barret's esophagus: increasing diagnostic yield. Am J Gastroenterol 2020;115:S201-S202.

18. Shaheen NJ, Falk GW, Iyer PG, Gerson LB. Corrigendum: ACG Clinical Guideline: diagnosis and management of Barrett's esophagus. Am J Gastroenterol 2016;111:1077.

19. Gross SA, Smith MS, Kaul V; US Collaborative WATS3D Study Group. Increased detection of Barrett's esophagus and esophageal dysplasia with adjunctive use of wide-area transepithelial sample with three-dimensional computer-assisted analysis (WATS).
United European Gastroent 2018;6:529-535.

20. Codipilly DC, Krishna Chandar A, Wang KK, et al. Wide-area transepithelial sampling for dysplasia detection in Barrett's esophagus: a systematic review and meta-analysis. Gastrointest Endosc 2021 Sep 17 [Online ahead of print]. doi: 10.1016/j. gie.2021.09.015

21. Singer ME, Smith MS. Wide area transepithelial sampling with computer-assisted analysis (WATS(3D)) is cost-effective in Barrett's esophagus screening. Dig Dis Sci 2021;66:1572-1579.

22. Docimo S Jr, Al-Mansour M, Tsuda S. SAGES TAVAC safety and efficacy analysis WATS(3D)(CDx Diagnostics, Suffern, NY). Surg Endosc 2020;34:3743-3747.

23. Amamra N, Touzet S, Colin C, Ponchon T. Current practice compared with the international guidelines: endoscopic surveillance of Barrett's esophagus. J Eval Clin Pract 2007;13:789-794. 